\title{
From Fuzzy Logic to Expert Systems*
}

\author{
BRIAN R. GAINES \\ and \\ MILDRED L. G. SHAW \\ Department of Computer Science, York University, \\ 4700 Keele Street, Downsview, Ontario, Canada M3J 1 P3
}

\begin{abstract}
The history and achievements of fuzzy set theory (FST) are considered in relation to developments in expert systems (ESs). An overview is given of early ES developments and the role of linguistic rules and metarules. It is suggested that FST and ESs are not just mathematical and technological advances but also represent major paradigm shifts in system theory. The main shift is away from the normative application of technology to change the world to be theoretically tractable, and towards increasing model realism. The limitations of classical system theory when applied to natural systems were the impetus behind the development of FST.
\end{abstract}

\section{INTRODUCTION}

Lotfi Zadeh first discussed the need for a "mathematics of fuzzy or cloudy quantities" in a paper entitled "From circuit theory to system theory" published in 1962. This led to his publishing his seminal paper, "Fuzzy sets," proposing such a mathematics in 1965. After two decades it is reasonable to look back and review the impact of this proposal. Has it led to a shift in our modes of thinking and problem-solving? Has it led to new perspectives on systems? Has it led to new tools? Is it being used in applications? Has the original purpose been achieved?

If one judges a concept by the activity it generates and the literature it generates, then that of fuzzy set theory (FST) must be rated very highly. A comprehensive bibliography for the first decade shows an increase from 2 papers published in 1965 to over 227 in 1975, with a cumulative total in 1975 of

* This paper is dedicated to Lotfi Zadeh, who has combined scientific work of the highest standard with tenacity, kindness, and patience. 
some 620 items [10] and in 1979 of some 1400 items [19]. The number of papers a year and cumulative total fit well to exponential growth at $60 \%$ a year for the first decade. However, it is now almost impossible to track the growth of a literature which as grown from the output of a small group of specialists to that of an international community involving almost every nation and discipline.

The rapid growth in the output of papers on FST during the decade from 1965 is the characteristic initial exponential form of the learning curve for a new discipline [4]. The overall form of the learning curve is logistic, with the initial exponential growth eventually becoming an exponential approach to an asymptotic output rate. Fitting empirical data to such a curve is notoriously sensitive to minor fluctuations [2]. The best fit to the 1965-1979 data gives an asymptote at 300 papers a year, with $10 \%$ of this being reached in $1970,50 \%$ in 1975 , and $90 \%$ in 1980. This suggests that the first half of the learning curve was the decade 1965-1975, and that we are now completing the second half in the decade 1975-1985. The 300 papers a year figure corresponds to about four times the output in the main journal on FST and is probably a realistic figure for refereed journal papers. The growth rate of the dissemination of knowledge about, interest in, and work on FST and its applications has been spectacular. There has been a shift in the modes of thinking and problem-solving for a significant community of theoretical and applied scientists and technologists.

In this paper we attempt to put this shift in perspective by examining the role of fuzzy reasoning in an applications area whose growth has itself been as spectacular, that of expert systems (ESs) and their applications [23, 15, 25]. This is an interesting area, not only for its high intrinsic value, but also because it enables us to contrast differing aspects of the role of FST in modern information science. Expert systems development leads to requirements for reasoning with imprecise data, where FST provides an alternative paradigm to those of classical logic and probability theory. The most well-recognized breakthroughs in ESs, such as MYCIN [28], were not based on FST, but on heuristic methods that turn out to resemble FST closely. Other early breakthroughs such as linguistic process controllers [20] were based directly on FST.

The next section gives an overview of early ES development commencing with control systems based on FST.

\section{EXPERT SYSTEMS: AN OVERVIEW}

The computer simulation of people in the roles of experts on some topic has become an important application of interactive computer systems. It has generated a new industry based on creating expert systems to make the practical working knowledge of a human expert in a specific subject area such as medicine or geology widely available to those without direct access to the 
original expert [25]. Programs now exist that have made practical achievements in medical diagnosis, interpretation of mass spectroscopy results, analysis of geological survey data, and other problems where one would normally go to a human expert for advice.

\section{EARLY EXPERT SYSTEMS}

One of the first ES developments was the fuzzy logic control system developed in 1974 by Mamdani and Assilian. The system accepted human knowledge of control strategies expressed verbally and encoded it directly as computer programs which acted on the environment [20]. This work was undertaken as part of a study of machine learning in process control, and the system controlled was a small steam engine. The verbal rules were of the form shown in Figure 1.

The history of this discovery that human control expertise could be expressed linguistically and encoded directly on a computer is itself interesting. The original nbjective of the work was to investigate learning controllers in a realistic situation, and the steam engine used was real, not a computer simulation. It was found that learning a control strategy from a zero base was problematic, since it did not happen, at least not before the steam engine ran out of water. Gaines and Andreae [9] had reported a similar problem with simulated control situations and introduced the notion of priming a learning machine with an initial suboptimal control strategy. Gaines [7] reported positive results with an adaptive threshold logic learning controller using a crude priming technique to simulate the effect of verbal instructions on a human trainee. Mamdani and Assilian decided to prime their system in a similar way, but using Zadeh's [36] suggestion of directly translating linguistic statements into decision rules using FST.

What was surprising at the time and made the 1974 results a recognized breakthrough was that the control rules derived from the verbal statements were extremely effective. They compared favorably with those derived by tuning a standard PID (proportional-integral-derivative) controller for optimum performance. Mamdani and Assilian also found that the learning machine then proceeded only to learn less effective strategies. Hence interest switched to the process whereby human expression of verbal rules that appear vague can lead to highly effective control strategies. In the past ten years Mamdani and Assilian's

\footnotetext{
If the pressure error is positive and big and the change in pressure error is not negative medium or big

Then make the heat change negative and big.
}

Fig. 1. Rule from fuzzy logic controller. 
1. When the burning zone temperature is drastically low:

(a) reduce kiln speed;

(b) reduce fuel.

2. When the burning zone temperature is slightly low:

(a) increase I.D. fan speed;

(b) increase fuel rate.

Fig. 2. Rules from a lime kiln operator's manual.

results have been replicated in many different countries for many different control processes, including a number of significant industrial processes such as pig iron smelting where effective automatic control had been thought to be impossible [21].

The concept of an ES was not prevalent at the time of the initial fuzzy control studies, and their significance as examples of early ES development was noted only later. It has also been noted that the transmission of decision and control knowledge through linguistic rules is a technique that has been used in the past quite independently of computer systems. Figure 2 is an excerpt from a training manual for lime kiln operators [24]. It seems that the encoding of expertise into a set of fairly vague but highly applicable rules is a standard way of making it available to others.

In parallel with the controller development, other rule-based ESs were being developed for completely different domains. The system widely recognized as an early breakthrough, MYCIN, is a medical diagnosis ES which aids a clinician to act as a consultant on infectious diseases [28]. It uses rulcs of the form shown in Figure 3. These rules are obtained from specialists in microbial infections, and their application to particular data is fairly simple data processing. The rules are validated through their application to many cases and revised when they fail to give the correct diagnosis. MYCIN is designed to interact with a clinician in order to make a diagnosis and suggest therapy for a particular patient with suspected microbial infections. It first gathers data about the patient and then uses them to make inferences about the infections and their treatment.

Note that the MYcIN rule of Figure 3 involves an assertion that is evidential rather than true. Shortliffe found it necessary to encode rules of inference that

\section{RULE 50}

If (1) the infection is primary-bacteremia, and

(2) the site of the culture is one of the sterile sites, and

(3) the suspected portal of entry of the organism is the gastrointestinal tract,

Then there is suggestive evidence (0.7) that the identity of the organism is bacteroides.

Fig. 3. A MYYIN rule. 
were imprecise and could not be encoded simply in terms of truth and falsity. He ascribed a verbal label, "suggestive evidence," and a numerical truth value, "0.7," to a rule and developed a calculus for combining such truth values in chains of logical inference. Thus, linguistic reasoning and multivalued logics were key components of early ES developments, although the MYCIN developers were initially unaware of FST and the linguistic controller developers were initially unaware of ES concepts.

Since the early success with MYCIN a very wide range of ESs have been developed. Gevarter [15] has summarized some well-known expert systems and their applications, but the numbers and domains have since increased so rapidly that it is now impossible to make any accurate count. Most universities have some activity in this field, and many industrial ESs are regarded as highly proprietary. The problem of extracting knowledge from experts and encoding it for computers is now termed knowledge engineering and is regarded as the major bottleneck in ES development [17]. Direct elicitation of a complete, noncontradictory set of linguistic rules from experts for a large domain has proved very difficult and time-consuming. However, Boose [3] has reported success in rapid prototyping of ESs for a large number of applications using knowledge engineering tools based on an FST model of personal construct psychology $[27,11]$.

\section{EXTENSION OF RULES TO METAKNOWLEDGE}

Mamdani, Procyk, and Baaklini [22] found that learning could be introduced effectively in the steam engine controller through metarules that expressed the way in which the basic rules should be changed as a result of performance feedback. The learning level of their controller operated on rules of the form shown in Figure 4. This was sufficient for the fuzzy controller to acquire a control strategy similar to that induced through verbal rules from a human expert.

Metarules were also introduced independently by Davis to aid the debugging of MYCIN. It was difficult to set up the MYCIN rules initially and also difficult to trace errors in the deductions. To overcome these problems TEIRESIAS [6] was added as an auxiliary ES with expertise about MYCIN to explain MYCiN's decisions and help the clinician amend the rules when they led to incorrect

\footnotetext{
If time is small and error is negative big

Then desired change is big.

If time is big and error is positive zero

Then desired change is zero.
}

Fig. 4. Metarules from fuzzy learning controller. 
METARULE003:

If (1) there are rules which do not mention the current goal in their premise

(2) there are rules which mention the current goal in their premise

Then it is definite that the former should be done before the latter.

Fig. 5. A teiresias metarule.

conclusions. TEIRESIAS uses a rule-based approach to reasoning like MYCIN's, but the rules are now rules about the forms of rules and the use of rules. A typical one of these metarules is shown in Figure 5.

Whereas MYcIN's rules are specific to microbial infections, those of TEIRESIAS are more general and can be used in other domains. Davis [5], for example, shows TEIRESIAS being used as an investment decision system for clients of a stockbroker.

One of the important features of MYCIN/TEIRESIAS that has become an essential characteristic of ESs is their capability to provide explanations of the deductions given. "Why?" questions are accepted as responses when data are requested, and are interpreted as a request for the rule to be shown that requires the data requested. A "why?" question may also be asked when conclusions are drawn, and is then interpreted as a request for the complete chain of logic used in arriving at that conclusion to be shown. The facility to answer such questions make ESs accountable for their behavior and conclusions. This is itself a major new feature of systems programmed for computers.

Another important feature of ESs is that they are not static representations of knowledge but can continue to acquire knowledge as they are used. Essentially, the use of metarules allows ESs to be programmed interactively by their users. From one perspective the metarules of the fuzzy learning controller and TEIRESIAS can be seen as an important development in automatic programming. From another they can be seen as a way in which a machine acquires knowledge through interaction with its environment or with a person. These are analogous to the fundamental ways in which people acquire knowledge [12]. For such applications computational logics capable of dealing with the uncertainties of imprecise data and fallible hypotheses are essential.

\section{SHIFTS IN SYSTEMS PARADIGMS}

The previous section has shown how the early applications of FST to control and decision systems paralleled the development of early expert systems in the use of linguistic rules, fuzzy reasoning, and metarules. This role of FST, significant as it is in itself, is only an indication of the deeper paradigm shifts from which FST and ESs both stem. The classical approach in decision and 
control system design is:

(1) thoroughly instrument the system to be controlled or about which decisions are to be made;

(2) use the instrumentation to gather data about the system behavior under a wide variety of circumstances;

(3) from these data build a model of the system that accounts for this behavior;

(4) from this model derive algorithms for decision or control that are optimal in terms of prescribed performance parameters.

This positivistic paradigm underlies the methodologies of the physical sciences and technologies based on them. It has the merit that it has been extremely successful in engineering much of the technological infrastructure of our current civilization.

However, this paradigm is successful only to the extent that the systems under consideration are amenable to instrumentation and modeling. Its greatest successes have been where this amenability can be achicved normativcly, that is, in cases where the system to be controlled is itself a human artifact. For example, linear system theory has not become a major tool in systems engineering because most natural systems are linear-they are not. The implication is in the opposite direction: that linear systems are mathematically tractable and that we design artificial systems to be linear so that we may model them readily.

The application of "a linear model with quadratic performance criterion" to natural systems is often attempted, but in general it does not work. We have done so not because the tool was appropriate, but because it was the only one we had. However, the use of a hammer to insert screws, although partially effective, tends to distort, destroy, and generally defeat the purpose of using a screw. Similarly, the use of an inappropriate system theory to model a system may give useful, but limited, results when we have no other, but it distorts reality, destroys information, and generally defeats the purpose of modeling that system.

Much of our current technology succeeds to the extent that it is normative. In agriculture we reduce the complexity of a natural ecology to a comprehensible simplicity by the use of pesticides, herbicides, and chemical fertilizers [13]. We reduce the system to one which is amenable to our modeling techniques. That simpler is not necessarily better and that reengineering nature to impose uniformity destroys variety which is itself valuable have only been realized in recent years.

The four shifts in perspective that we see in FST and ESs are:

Problem 1. The models available are inadequate to capture the system.

Old approach: Procrustean design. Change the world to fit the model-normative technology. 
Paradigm shift: Model realism. Use system methodologies and information technology that enable the natural world to be modeled without distortion and destruction.

Problem 2. Optimal control is oversensitive to system uncertainties.

Old approach: Suboptimality. Use a suboptimal controller that is robust.

Paradigm shift: Model uncertainty. Model the uncertainty as part of the system.

Problem 3. Data are unavailable or inadequate for modeling.

Old approach: Managing. Do not automate-leave to human decision/control.

Paradigm shift: Expert systems. Model the person as a decision-maker or controller.

Problem 4. Neither a human nor an automatic system alone is adequate.

Old approach: Ad hoc system design. Use a mixture of automatic and human decision/control.

Paradigm shift: Accountable integration. Integrate automatic and human activity - make the automation accountable ("why?" in ESs).

The last three perspectives all stem from the first. The importance of this first perspective to Zadeh is apparent in his 1962 paper, where he discusses the fundamental inadequacy of conventional mathematics for coping with the analysis of biological systems, noting also that the need for a new mathematics was becoming increasingly apparent even in the realm of inanimate systems.

The second perspective is that which led to FST. Optimal control theory was regarded as the peak achievement of system theory in the 1950s and 1960s. However, it proved limited in application because it demanded precision in system modeling that was impossible in practice. It was too sensitive to the nuances of system structure expressed through overprecise system definition.

The third perspective is that which led to the success of linguistic fuzzy controllers and later ESs. Hayes-Roth [16] has noted the many problems that have been felt to require human management are now amenable to ESs. Modeling the way the expert performs the task rather than modeling the task itself is the primary characteristic of an ES.

The fourth perspective is an important one for both ESs and FST. They are knowledge-based systems because they make provision for explaining the decisions reached in terms of the data and inferences used. It is interesting to note that logics of uncertainty that aggregate evidence, such as probabilistic logics, do not provide a simple mechanism for explanation. Explicable logics have to be truth-functional and nonaggregative; fuzzy logic satisfies these requirements (uniquely among those logics satisfying the weak axioms of a standard uncer- 
tainty logic [8]). It is also interesting to note that the capability to give explanations is seen by some philosophers of science as a key difference between models that make accurate predictions and scientific theories that in addition provide causal explanations [26]. The "why?" question has important implications for both the practical and theoretical significance of ESs and the logics on which they are based.

\section{ZADEH'S ROLE IN THE PARADIGM SHIFTS}

The development of FST and ESs is not just a technological improvement but represents a major paradigm shift in system theory. Zadeh was ideally placed to understand the strengths and weaknesses of system theory, and the significance of developments in artificial intelligence. The sixty-five papers he published in the fifteen years preceding "Fuzzy sets" [35] represent the peak achievements of mathematical system theory on a broad front. One of his earliest papers is on thinking machines as a new field in electrical engineering [29]. His technical papers commence with filtering and prediction techniques at the frontiers of linear system theory, for example on linear time-varying systems. Many of the papers contain the term "general" in their title, and many of them are applied to circuit and control systems. Zadeh's aspirations were clearly defined from the start: to develop general mathematical theories that could be applied in engineering design.

In the later papers of that era Zadeh grapples with the problem of formulating engineering concepts whose intuitive significance is of paramount important but which are difficult to encompass theoretically. He discusses the "identification problem" [30], "what is optimal" [31], "the definition of adaptivity" [33], and the "concept of state" [34]. These papers represent the most advanced system thinking of that era and were seminal in content. They were based on classical mathematical system theory and widely recognized as breaking new ground. It was a shock for many when Zadeh turned away from this work after 1965 and began to develop and promote first the concepts of FST and second its instantiation through fuzzy linguistic reasoning.

FST was from the outset an attempt to create a new mathematical system theory that corresponds to paradigm shift 1 and fits the realities of the world without distorting them. It was created by a person who had extended the boundaries of current system theory, attempted to encompass in generality the key concepts of applied systems engineering, and recognized the failure of that theory in this task. FST was also highly controversial from the start and aroused strong emotional responses. Kalman [18] comments on an FST paper by Zadeh:

His proposals could be severely, ferociously, even brutally criticized from a technical point of view.... No doubt Professor Zadeh's enthusiasm for fuzzy sets has been reinforced by the prevailing political climate in the U.S.: one of unprecedented permissiveness. 
Arbib [1], in a book review, is more gentle in his criticism but casts doubts on the appropriateness of FST ("A horrifying thought-what if Newton had rejected the concept of mass, and sought to base his theory on a 'degree of heaviness' between 0 and 1 for each object in the universe?") and chides those writing on FST for their insularity in not linking it with existing material on multivalued logics, linguistic semantics, and so on.

Criticism and controversy are the hallmarks of significant paradigm changes and key components of the process of scientific development and progress. So is the accompanying literature explosion, much of which is inadequately founded due to the inexperience of not only authors but also referees in the material of the new paradigm. Those days are now past. The key journals on FST are well refereed, the mathematical foundations are solid, and the many links with related topics and literature have been well documented. Against the background of ESs, human reasoning processes are now treated with greater respect and linguistic reasoning is being widely investigated. The notion of "degree of heaviness" of an object does precede that of its mass, and the development of naive physics on the basis of such informal concepts is now regarded as a major challenge for artificial intelligence and cognitive science research [14].

\section{CONCLUSIONS}

After two decades of fuzzy set theory, with the development of mathematical foundations and a wealth of applications, it is easy to look back and wonder what all the controversy was about. We have suggested that FST and ESs, and the application of one to the other, are not just mathematical and technological advances but also represent major paradigm shifts in system theory. The controversy has been about quite fundamental changes in system philosophy and technology, shifts from a positivistic, normative approach to a more realistic and naturalistic approach. These shifts are apparent throughout science and technology and its application to our world and society.

Lotfi Zadeh recognized the changes necessary in system theory at an early stage and pioneered in developing new approaches. The technical success of his work and the wide-ranging theoretical and practical advances stemming from it are tributes in themselves. However, technical tributes alone are not enough to indicate the courage needed to drop well-established lines of research and swim against the current of scientific thought. His perseverance in the face of criticism, his patient explanations, his kindness in supporting others, and his willingness to present the material to unsympathetic audiences, make our tributes as much personal as technical.

Fuzzy set theory cannot be either right or wrong. It is applicable mathematics tested by its uses. However, the rationale behind it, the systemic principles 
involved, can be right or wrong. They are right for our time, for the objectives of dealing adequately with a complex universe and extending the capabilities of the person with computer enhancements. The redevelopment of system theory is not yet complete, and the seminal notions of stability, adaptivity, modeling, and so on still need adequate expression. However, we now have the foundations on which to build a system theory that combines realism with power and provides applicable mathematics for our knowledge-based society.

Financial assistance for this work has been made available by the National Sciences and Engineering Research Council of Canada.

\section{REFERENCES}

1. M. Arbib, Book review of applications of fuzzy sets to systems analysis, $J$. Soc. Industrial and Appl. Math. 19:753 (1977).

2. W. Ascher, Forecasting: An Appraisal for Policy-Makers and Planners, Johns Hopkins U.P., Baltinore, 1978.

3. J. H. Boose, Personal construct theory and the transfer of human expertise, in Proceedings AAAI-84, Amer. Assoc. for Artificial Intelligence, Calif., 1984, pp. 27-33.

4. D. Crane, Invisible Colleges: Diffusion of Knowledge in Scientific Communities. Univ. of Chicago Press, Chicago, 1972.

5. R. Davis, TEIRESIAS: Experiments in communicating with a knowledge-based system, in Designing for Human-Computer Communication (M. E. Sime and M. J. Coombs, Eds.), Academic, London, 1983, pp. 87-137.

6. R. Davis and D. B. Lenat, Knowledge-Based Systems in Artificial Intelligence, McGraw-Hill, New York, 1982.

7. B. R. Gaines, The learning of perceptual-motor skills by men and machines and its relationship to training, Instructional Sci. 1(3):263-312 (Oct. 1972).

8. B. R. Gaines, Precise past-fuzzy future, Internat. J. Man-Machine Stud. 19(1):117-134 (July 1983).

9. B. R. Gaines and J. H. Andreae, A learning machine in the context of the general control problem, in Proceedings of the 3rd Congress of the International Federation for Automatic Control, Butterworths, London, 1966.

10. B. R. Gaines and L. J. Kohout, The fuzzy decade: A bibliography of fuzzy systems and closely related topics, Internat. J. Man-Machine Stud. 9(1):1-68 (Jan. 1977).

11. B. R. Gaines and M. L. G. Shaw, Logical foundations of expert systems, in Proceedings of IEEE International Conference on Systems, Man and Cybernetics, Halifax, Nova Scotia, Oct. 1984, pp. 238-247.

12. B. R. Gaines and M. L. G. Shaw, The Art of Computer Conversation: A New Medium for Communication, Prentice-Hall, Englewood Cliffs, N.J., 1984.

13. B. R. Gaines and M. L. G. Shaw, Expert systems: The substitution of knowledge for materials in world food production, Possible Worlds 1(2):5-8 (July 1984),

14. D. Gentner and A. L. Stevens, (Eds.), Mental Models, Lawrence Erlbaum, Hillsdale, N.J., 1983.

15. W. B. Gevarter, Expert systems: Limited but powerful, IEEE Spectrum 18:39-45 (1983).

16. F. Hayes-Roth, The industrialization of knowledge engineering, in Artificial Intelligence Applications for Business (W. Reitman, Ed.), Ablex, Norwood, N.J., 1984, pp. 159-177. 
17. F. Hayes-Roth, D. A. Waterman, and D. B. Lenat (Eds.), Building Expert Systems, Addison-Wesley, Reading, Mass., 1983.

18. P. J. Kalman, Discussion of "A new approach to system analysis" by L. A. Zadeh, in Man and Computer (M. Marois, Ed.), North-Holland, New York, 1974, p. 93.

19. A. Kandel and R. R. Yager, A 1979 bibliography of fuzzy sets, their applications and related topics, in Advances in Fuzzy Set Theory and Applications (M. M. Gupta, R. K. Ragade, and R. R. Yager, Eds.), North-Holland, Amsterdam, 1979, pp. 621-744.

20. E. H. Mamdani and S. Assilian, An experiment in linguistic synthesis with a fuzzy logic controller, in Fuzzy Reasoning and its Applications (E. H. Mamdani and B. R. Gaines, Eds.), Academic, London, 1981, pp. 311-323.

21. E. H. Mamdani, J. J. Ostergaard, and E. Lembessis, Use of fuzzy logic for implementing rule-based control of industrial processes, in Fuzzy Sets and Decision Analysis (H. J. Zimmermann, L. A. Zadeh, and B. R. Gaines, Eds.), TIMS Series Studies in the Management Sciences, 20, North-Holland, Amsterdam, 1984.

22. E. H. Mamdani, T. Procyk, and N. Baaklini, Application of fuzzy logic to controller design based on linguistic protocol, in Discrete Systems and Fuzzy Reasoning (E. H. Mamdani and B. R. Gaines, Eds.), Queen Mary College, London, 1976, pp. 125-149.

23. D. Michie (Ed.), Expert Systems in the Micro Electronic Age, Edinburgh, U.P., Edinburgh, 1970.

24. K. E. Peray and J. J. Waddell, The Rotary Cement Kiln, Chemical Publishing, New York, 1972.

25. W. Reitman (Ed.), Artificial Intelligence Applications for Business, Ablex, Norwood, N.J., 1984.

26. W. Salmon, Scientific Explanation and the Causal Structure of the World, Princeton U.P., Princeton, N.J., 1984.

27. M. L. G. Shaw, Interactive knowledge elicitation, in Proceedings of CIPS Session 84, Canad. Inform. Processing Soc., Calgary, May 1984, pp. 202-208.

28. E. H. Shortliffe, Computer-Based Medical Consultations: MYcIN, Elsevier, New York, 1976.

29. L. A. Zadeh, Thinking machines-a new field in electrical engineering, Columbia Engrg. Quart. 3:12-13 (1950).

30. L. A. Zadeh, On the identification problem, IRE Trans. Circuit Theory CT-3:277-281 (1956).

31. L. A. Zadeh, What is optimal?, IRE Trans. Circuit Theory CT-4:3 (1957).

32. L. A. Zadeh, From circuit theory to system theory, Proc. Inst. Radio Engrs. 50:856-865 (1962).

33. L. A. Zadeh, On the definition of adaptivity, Proc. IEEE 51:469-470 (1963).

34. L. A. Zadeh, The concept of state in system theory, in Proceedings of the Second Systems Symposium, Wiley, New York, 1964.

35. L. A. Zadeh, Fuzzy sets, Inform. and Control 8:338-353 (1965).

36. L. A. Zadeh, Outline of a new approach to the analysis of complex systems and decision processes, IEEE Trans. Systems Man Cybernet. SMC-2:28-44 (1973). 